IIUC STUDIES

ISSN 1813-7733

Vol.- 7, December 2010

(Published in December 2011) (p 241-248)

\title{
Power System Switching Transient Detection using Wavelet Transformed Based Signal Decomposition
}

\author{
M. Shafiul Alam* \\ Md. Shamimul Haque Chowdhury ${ }^{* *}$ \\ Muhammad Athar Uddin ${ }^{*}$
}

\begin{abstract}
Switching transient phenomena in Electric Power System develop several disturbances, sometimes very hazardous for the electrical equipment life, for the environment and for the human life. Switching transient phenomena produce over voltage, over current and electrical fields, which haven't to neglect. Several types of wavelet network algorithms have been considered for detection of power system switching transients. But both time and frequency information are accessible by multiresolution analysis (MRA). This paper presents a wavelet transform based multiresolution analysis of power system signal to detect, localize and extract switching transients. Power system switching transients have been simulated using MATLAB-7.01. The key idea underlying the approach is to decompose a distorted signal into other signals which represents a smoothed version and detailed version of the original signal. The decomposition is performed using multiresolution analysis. The proposed method appears to be robust for detection and localization of power quality disturbances produced due to load switching and capacitor switching.
\end{abstract}

Key words: Power system, switching transient, wavelet transform, multiresolution analysis (MRA), signal decomposition.

\section{Introduction}

Wavelet Transform has achieved immense attention during the last several years, because it is better suited for the analysis of certain types

* Lecturer, Dept. of Electrical \& Electronic Engineering, IIUC, Dhaka Campus.

** Lecturer, Dept. of Electrical \& Electronic Engineering, IIUC, Dhaka Campus.

*** Assistant Professor, Dept. of Electrical \& Electronic Engineering, IIUC, Dhaka Campus. 
of transient waveforms than the other transforms approach like Fourier transform and short time Fourier transform. Several works have been developed in many areas with the aim of this tool especially in the last few years as these have met the potential benefits of applying wavelet transform due to superior analyzing and processing of the voltage/current signals in order to make a real time identification of transients. In 2000, Styvaktakis, et al.[1] presented new algorithm for identification and classification of transients due to synchronized capacitor switching in three phase systems. The algorithm is tested using measurements performed at $400 \mathrm{~V}$ and $10 \mathrm{kV}$ by Goteborg in Sweden. Zero-crossing and ' $5 \mathrm{msec}$ ' capacitor energizing are considered. The classification is based on the withdrawal of the point of the wave on the phase to ground voltage waveforms where the switching of each phase takes place. Discrete Wavelet Transform (DWT) is used for this purpose. In 2000, Fernando H. Magnago, Ali Abur [2], presented a wavelet based approach to model frequency dependent lines. The method uses the Bergeron's traveling wave model. Instead of using the convolution procedure, multiresolution decomposition of the wavelet transform is applied for transient analysis. In this case the parameters are assumed constant at each scale of the wavelet transform. Accuracy of this assumption improves with proper choice of scales. In 2009, Rodney H.G. Tan and V.K. Ramachandaramurthy [3], presented power system transient analysis using scale selection wavelet. The scale selection wavelet transform is carried out by performing continuous wavelet transform on the signal of interest at selected scales ranging from 1 to 70 . The standard deviation of the wavelet scales energy is calculated to distinguish impulsive and oscillatory transient disturbances. Finally in 2010, Gilany, et al. [4], presented a wavelet based technique for finding and categorization of unusual conditions that occur on power distribution lines. The proposed method depends on the sensitive fault detection (SFD) parameter calculated through wavelet multiresolution decomposition. The use of wavelet transform with the proposed SFD parameter makes it easy not only to detect the occurrence of a fault and its type but also to make a distinction between transients due to switching conditions and that due to faults.

In this paper we present a wavelet transform based multiresolution signal decomposition to detect and localize power system switching transients in time domain. The basic idea is to decompose a given distorted signal into other signals that present a smoothed version and detailed version of original signal. The detailed version is really a 
wavelet transforms of original signal that shows the occurrence of disturbance event, frequency content of the event, and the gradient of the event.

\section{Introduction to wavelet}

Wavelets are mathematical functions that cut up data into different frequency components and then study each component with a resolution matched to its scale. Wavelet, as its name suggests "small wave" which have finite energy that integrates to zero. It employs a prototype function called the mother wavelet. This function sharply decays in an oscillatory fashion. Fourier analysis consists of breaking up a signal into sine waves of various frequencies. On the other hand wavelet analysis is the breaking up of a signal into shifted and scaled versions of the original (or mother) wavelet.

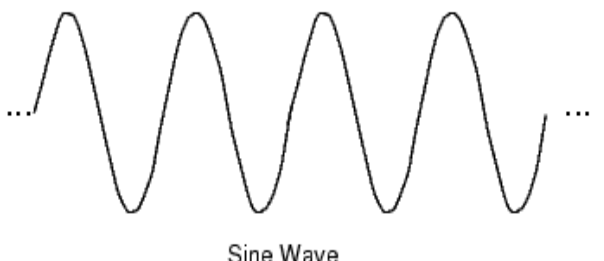

Figure:1 Sine wave and wavelet

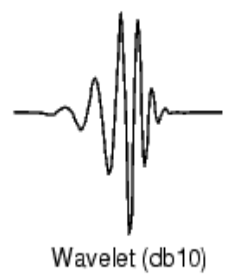

Wavelet (db10)

Circumspectly looking at Fig. 1 of wavelet and sine wave, we can see that signals with quick changes might be better analyzed with an irregular wavelet than with a smooth sinusoid; just as some foods are superior handled with a fork than a spoon. The local features can be explained better with wavelets that have local extent. Wavelet analysis provides the time scale analysis of non-stationary signal [5]. It decomposes the signal into time scale rather than time frequency representation. Wavelet transforms acts in response to the gradient of a given signal. The more irregular the disturbance, the higher the gradient will be. In the power system signals, the wave shape of the disturbance occurrence is irregular compared to that of its background signal. So the wavelet coefficients associated with disturbance will have very large magnitudes compared to those of disturbance free waveform which will efficiently help to identify any disturbance event like switching transient. There are many wavelet families. The choice of analyzing wavelets plays a significant role in detection and localizing various types of power quality disturbances. As per IEEE standards, daubechies wavelet transform is very perfect for analyzing 
power quality disturbances. The names of the Daubechies family wavelets are written $\mathrm{dbN}$, where $\mathrm{N}$ is the order, and $\mathrm{db}$ the "surname" of the wavelet. Daubechies family wavelets are shown below-

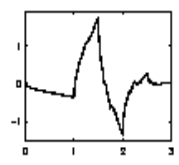

db2

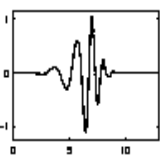

$\mathrm{db} 7$

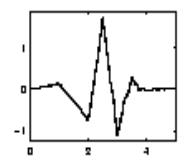

$\mathrm{db} 3$

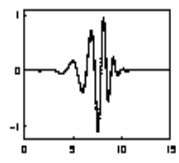

$\mathrm{db} 8$

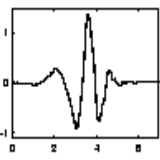

$\mathrm{db} 4$

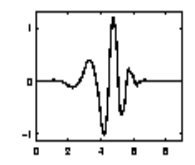

db5

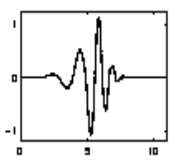

db6

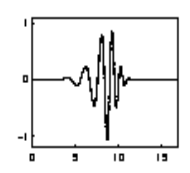

dbg

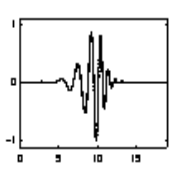

db10

Figure. 2: Daubechies wavelets family

\section{Multiresolution analysis using wavelet filter bank}

Wavelet analysis can be out looked as passing an input signal through a filter bank with variable central frequency and bandwidth [6]. The coefficients of such filter bank are calculated by the selected mother wavelet [7-8]. Filter bank is composed of multi stages, each stage is composed of low pass filter(LPF) and high pass filter(HPF) with the output approximation and detail respectively [7]. This filter structure can create a multiresolution analysis[7-8].

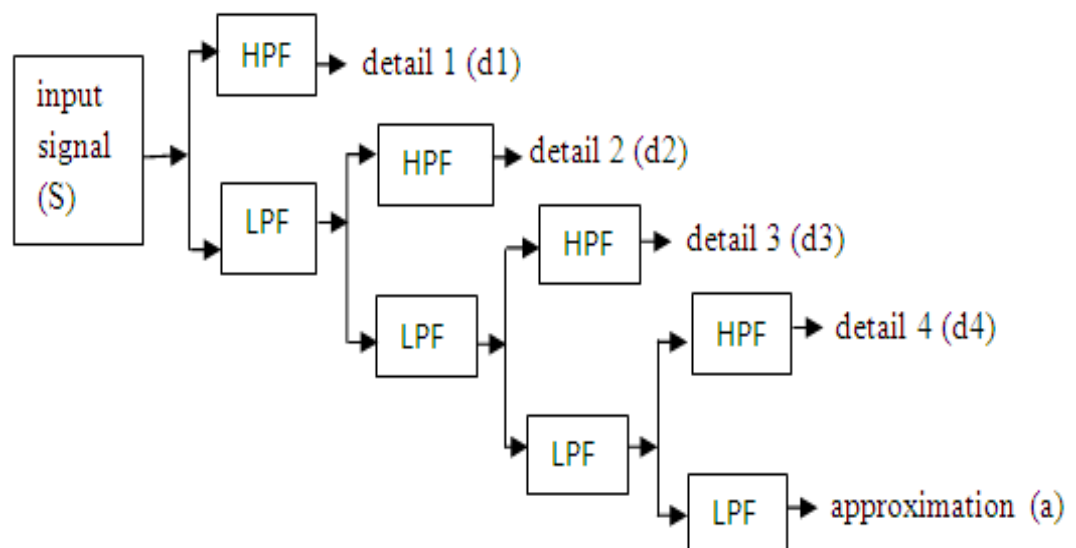

Figure: 3 Four level multiresolution signal decomposition using wavelet filter bank 


\section{Simulation results for power system switching transient detection and localization}

MATLAB simulink toolbox is used to model a simple power system with a generator(200 MW), and a load(200 MW) at a distance $150 \mathrm{~km}$ from the generator. Transmission line is presented with pi model. Load switching and capacitor switching transient faults on this simple power system are applied sequentially and these distorted signals are analyzed using MATLAB wavelet toolbox. Fig. 2 represents the test system.

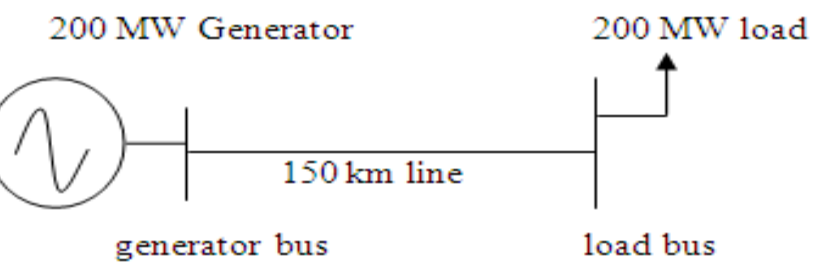

Figure. 4 Test power system

\section{A. Load switching transient detection and localization}

Load switching transient is applied to the test power system using a breaker connected before the load. Load is continuously taking power from generator. Now suddenly breaker is switched off and switch on at time $0.08 \mathrm{sec}$ and $0.14 \mathrm{sec}$ respectively to produce load switching transient. Fig. 5 shows distorted voltage waveform due to load switching transient.

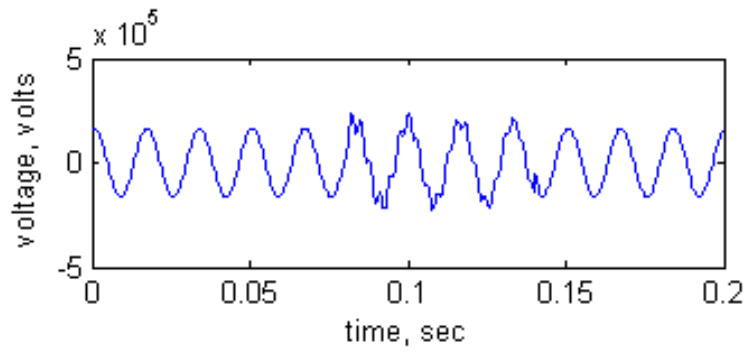

Figure. 5 Load switching transient signal

Now the distorted voltage waveform is decomposed through the wavelet filter bank. The wavelet transform filter bank acts in response most to the gradient of a given signal. The more irregular the disturbance, higher the gradient will be. In most power signals, the wave shape of disturbance event is irregular compared to that of its 
background signal. As a result, the wavelet coefficients related with the disturbance event will have very large magnitude compared to its disturbance free background signal [9]. Squared detailed signal at first level decomposition clearly detects and localizes occurrence of load switching transient in time domain. Two spikes of squared detailed signal effectively show occurrence of load switching.
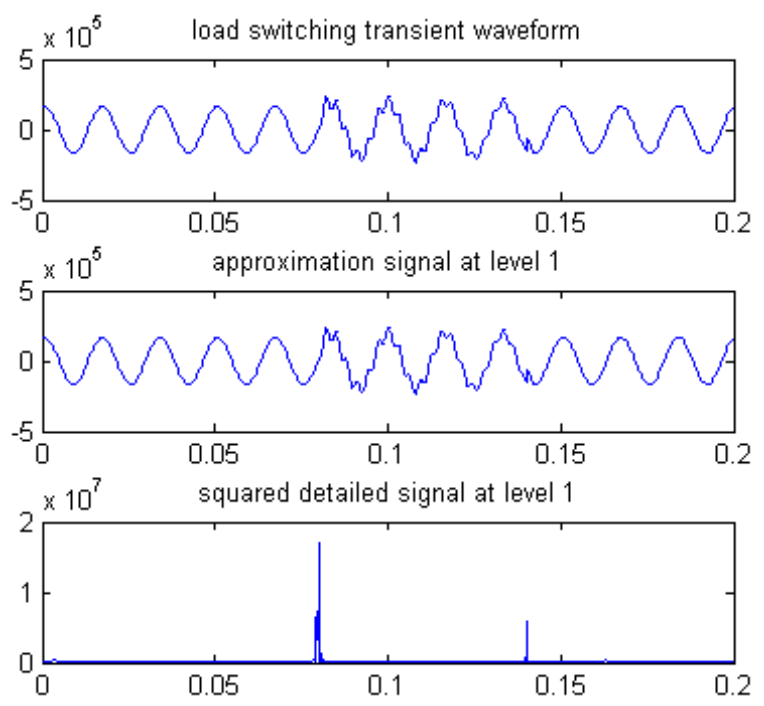

Figure: 6 Load switching transient detection and localization using squared detailed signal

\section{B. Capacitor switching transient detection and localization}

Fig. 7 shows the voltage waveform due to capacitor switching disturbance event. An $8 \mu \mathrm{f}$ capacitor is switched at $0.14 \mathrm{sec}$. on the test power system.

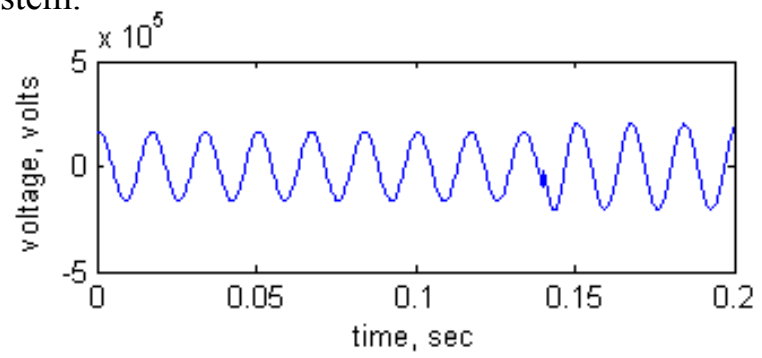

Figure:7 Capacitor switching transient signal 
Now multiresolution decomposition is performed on this capacitor switching transient waveform using wavelet transform filter bank. Squared detailed signal at first level decomposition clearly detects and localizes occurrence of capacitor switching transient in time domain. A large spike of squared detailed signal effectively shows occurrence of capacitor switching transient.
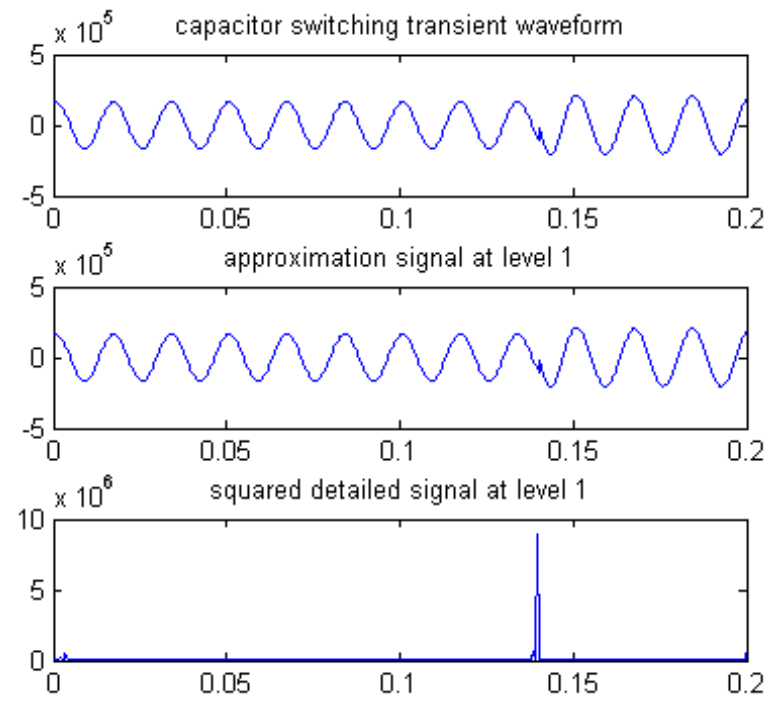

Figure:8 Capacitor switching transient detection and localization using squared detailed signal

\section{Conclusion}

This paper presents a wavelet transform based signal decomposition to detect and localize various types of electric power quality disturbances like load switching and capacitor switching transients. The power system disturbance signals are irregular compared to its background waveforms. Power system disturbance signals are decomposed into two versions i.e. the smoothed and detailed versions. The detailed versions essentially present the high frequency components of the disturbance signal. The squared detailed signals carry important features of the disturbance signals which effectively detect and localize power quality switching transients. The simulation results show powerful capabilities of the proposed method. 


\section{References:}

1 EMMANOUIL STYVAKTAKIS, MATH H.J. BOLLEN, IRENE Y.H. GU, "Classification of Power System Transients: Synchronized Switching”, IEEE Power Engineering Society Winter Meeting, 2000, Vol.4,pp.2681-2686.

2 FERNANDO H. MAGNAGO, ALI ABUR, "Wavelet-Based Simulation of Transients Along Transmission Lines With Frequency Dependent Parameters" IEEE Power Engineering Society Summer Meeting, 2000, Vol.2, pp.689-694.

3 RODNEY H.G. TAN, V.K. RAMACHANDARAMURTHY, "Power System Transient Analysis Using Scale Selection wavelet Transform”, IEEE Region 10 Conference, TENCON 2009, pp-1-6.

4 M. GILANY, N. ZAMAN, WAEL WAHABA, "A Wavelet Based Technique for Detection and Classification of transients in Distribution Networks", The 2nd International Conference on Computer and Automation Engineering (ICCAE), 2010, Vol-5, pp-118-122.

5 S. SANTOSO, E.J. POWERS, W.M. GRADY, P. HOFMANN, "Power Quality Assessment Via WaveletTransform Analysis", IEEE transactions on power delivery, Vol. 11, No. 2, pp. 924-930, April 1996.

6 CHUI, C.K., "WAVELET: A MATHEMATICAL TOOL FOR SIGNAL PROCESSING", Society for industrial and applied mathematics, Philadelphia, 1997.

7 ZHUANG, Y. AND BRASS, J.S.,"optimal wavelet basis selection for signal representation",CSHCN T.R.94-7, Centre for satellite and hybrid communication networks, 1994.

8 STANG, G. AND NGUYEN, T.,"wavelet and filter banks", WelleslyCambridge Press, 1996.

9 S. SANTOSO, E.J. POWERS, W.M. GRADY, "power quality disturbance identification using wavelet transforms and artificial neural networks" IEEE proceedings of the international conference on harmonics and quality of power-VII. Las Vegas, NV, pp. 615-618, October 1996. 